\title{
Can Smartphone-Derived Step Data Predict Laboratory-Induced Real-Life Like Fall-Risk in Community- Dwelling Older Adults?
}

\author{
Yiru Wang ${ }^{1 \dagger}$, Rachana Gangwani ${ }^{1,2+}$, Lakshmi Kannan ${ }^{1,3}$, Alison Schenone ${ }^{1}$, \\ Edward Wang ${ }^{1}$ and Tanvi Bhatt ${ }^{1 *}$
}

${ }^{1}$ Department of Physical Therapy, College of Applied Health Sciences, University of Illinois at Chicago, Chicago, IL, United States, ${ }^{2}$ MS Program in Rehabilitation Sciences, Department of Physical Therapy, College of Applied Health Sciences, University of Illinois at Chicago, Chicago, IL, United States, ${ }^{3}$ Ph.D. Program in Rehabilitation Sciences, Department of Physical Therapy, College of Applied Health Sciences, University of Illinois at Chicago, Chicago, IL, United States

OPEN ACCESS

Edited by:

Sjoerd M. Bruijn,

VU University

Amsterdam, Netherlands

Reviewed by:

Gaspar Epro,

London South Bank University,

United Kingdom

Chesney Elizabeth Craig,

Manchester Metropolitan University,

United Kingdom

*Correspondence:

Tanvi Bhatt

tbhatt6@uic.edu

${ }^{\dagger}$ These authors share first authorship

Specialty section:

This article was submitted to Biomechanics and Control of Human

Movement,

a section of the journal

Frontiers in Sports and Active Living

Received: 25 March 2020 Accepted: 20 May 2020

Published: 10 July 2020

Citation:

Wang Y, Gangwani R, Kannan L, Schenone A, Wang $E$ and Bhatt $T$ (2020) Can Smartphone-Derived Step

Data Predict Laboratory-Induced Real-Life Like Fall-Risk in Community-Dwelling Older Adults?

Front. Sports Act. Living 2:73. doi: 10.3389/fspor.2020.00073
Background: As age progresses, decline in physical function predisposes older adults to high fall-risk, especially on exposure to environmental perturbations such as slips and trips. However, there is limited evidence of association between daily community ambulation, an easily modifiable factor of physical activity (PA), and fall-risk. Smartphones, equipped with accelerometers, can quantify, and display daily ambulation-related PA simplistically in terms of number of steps. If any association between daily steps and fall-risks is established, smartphones due to its convenience and prevalence could provide health professionals with a meaningful outcome measure, in addition to existing clinical measurements, to identify older adults at high fall-risk.

Objective: This study aimed to explore whether smartphone-derived step data during older adults' community ambulation alone or together with commonly used clinical fall-risk measurements could predict falls following laboratory-induced real-life like slips and trips. Relationship between step data and PA questionnaire and clinical fall-risk assessments were examined as well.

Methods: Forty-nine community-dwelling older adults (age 60-90 years) completed Berg Balance Scale (BBS), Activities-specific Balance Confidence scale (ABC), Timed Up-and-Go (TUG), and Physical Activity Scale for the Elderly (PASE). One-week and 1-month smartphone steps data were retrieved. Participants' 1-year fall history was noted. All participants' fall outcomes to laboratory-induced slip-and-trip perturbations were recorded. Logistic regression was performed to identify a model that best predicts laboratory falls. Pearson correlations examined relationships between study variables.

Results: A model including age, TUG, and fall history significantly predicted laboratory falls with a sensitivity of $94.3 \%$, specificity of $58.3 \%$, and an overall accuracy of $85.1 \%$. Neither 1-week nor 1-month steps data could predict laboratory falls. One-month steps data significantly positively correlated with BBS $(r=0.386, p=0.006)$ and ABC $(r=$ $0.369, p=0.012)$, and negatively correlated with fall history $\left(r_{p}=-0.293, p=0.041\right)$.

Conclusion: Older participants with fall history and higher TUG scores were more likely to fall in the laboratory. No association between smartphone steps data and laboratory 
fall-risk was established in our study population of healthy community-dwelling older adults which calls for further studies on varied populations. Although modest, results do reveal a relationship between steps data and functional balance deficits and fear of falls.

Keywords: fall prediction, steps data, smartphone technology, falls, older adults

\section{INTRODUCTION}

Falls are a common and a serious problem in older adults aged 60 years and above (Rubenstein, 2006; Carpenter et al., 2019). Even the healthiest community-dwelling older adults are not immune to falls, especially on exposure to external environmental perturbations such as slips and trips which accounts for $60 \%$ of outdoor falls among community-dwelling older adults aged 70 years and above (Berg et al., 1997; Luukinen et al., 2000; Crenshaw et al., 2017). Such falls occur due to agerelated physiological changes resulting in balance dysfunction, reduced muscle strength, and impaired gait pattern, predisposing older adults to high fall-risk (Ambrose et al., 2013; Zhao et al., 2018). Additionally, with progressing age, older adults experience a decline in physical function and activities of daily living which further increases fall-risk (Smee et al., 2012; Welmer et al., 2017). Falls result in several deleterious physical consequences such as fractures and soft tissue injuries but also lead to fear of falling, thereby resulting in further self-imposed restriction of physical activity (PA) (Pereira et al., 2014; Young and Mark Williams, 2015).

Apart from aging, a fall-risk factor which is non-modifiable, PA which can mitigate age-related declines in muscle strength, balance, and agility is considered a modifiable risk-factor. Due to its adaptability, PA could be systematically monitored and enhanced in the community-living geriatric population to reduce fall-risk. Although sparse, evidence using the selfreported questionnaire, Physical Activity Scale for the Elderly (PASE), demonstrated that fallers had lower PASE scores (less PA) compared to non-fallers and that the low scores were associated with high fall-risk and fear of falling (Roig et al., 2011; Oliveira et al., 2017). Such a questionnaire-based assessment can serve as an inexpensive tool to assess PA among community-dwelling older adults over a period of 7 days (Washburn et al., 1993; Logan et al., 2013; Duray and Genc, 2017). However, it has several limitations. Firstly, self-reported techniques show recall bias, especially when used by older adults with possibly declining memory. Additionally, the need to give socially desirable answers can affect the accuracy of results (Perell et al., 2001). Finally, studies suggest that PASE might have a floor effect because several activities listed in PASE, such as outdoor gardening, yard work, painting, and wall papering, might not be commonly performed activities by older adults (Sallis and Saelens, 2000).

Contrary to self-reported measures, wearable sensor technology comprising of research-based (ActivPal and ActiGraph) and commercially available (FitBit and Apple watch) motion sensors automatically track and store PA and thus effectively combat the issue of recall-bias. Such accelerometer-based wearable sensors are able to identify PA patterns (frequency, duration, and intensity) under both controlled laboratory conditions and uncontrolled, realistic conditions of daily living (Plasqui and Westerterp, 2007; Gomersall et al., 2016; Rosenberger et al., 2016). Additionally, commercially available wearable sensor technology records and stores PA simply in terms of number of steps for several months and years. Thus, commercially available wearable sensors have gained popularity for PA monitoring in both young and older adult populations. The advantage of such technology is that step count can be easily interpreted by older adults themselves or by clinicians and used for comparative analysis by researchers. Among various PA parameters (distance covered, number of steps, and energy expenditure), step data has become the hallmark measure of PA monitoring (Tudor-Locke et al., 2005). One probable reason that steps are being used to represent PA is because walking is one of the most commonly reported forms of activity performed even among sedentary older adults (Paillard et al., 2004). In addition, other than its significant health benefits, walking has become a focus for public health interventions because of its feasibility and acceptability (Li et al., 2005).

Although commercially available accelerometer-based wearable sensors have several advantages, they have demonstrated reduced long-term compliance in older adults (compared to the younger population) due to the need of carrying an extra device and sometimes the cost associated with it (Marschollek et al., 2011; Ferrari et al., 2012). Thus, with the advancement in smartphone technology, the latter have replaced or supplemented wearable sensors for PA monitoring. Smartphones equipped with tri-axial accelerometers can be used in conjunction with inbuilt or freely available smartphone applications for measuring and recording step data. Studies indicate that smartphone applications similar to wearable sensor technology can deliver step data in a user-friendly interface (Higgins, 2016; Lu et al., 2017). For example, Harries et al. reported that participants had greater adherence to using the smartphone applications than wearing their wearable devices because running the smartphone application was quite simple and did not require much effort (Harries et al., 2016). In addition to their easy implementation, studies demonstrated that the accuracy of step data collected using smartphones was just as high as accelerometer-based sensors (Higgins, 2016; Lu et al., 2017).

Although the significance of $\mathrm{PA}$ as a modifiable fallrisk factor is established (Chan et al., 2007; Pereira et al., 2008), to the authors' knowledge there is limited evidence of association between daily life ambulation and fall-risk. Studies that demonstrated the association of daily-life ambulation and 
fall-risk (Rispens et al., 2015; van Schooten et al., 2015), utilized wearable-sensor technology and determined various daily-life ambulatory gait parameters that can predict fall-risk in older adults. However, they did not take number of steps into consideration. Limited studies have considered using number of steps as a parameter to identify fallers. Brodie et al. (2015) found that shorter ambulatory periods with fewer steps recorded using a wearable sensor can identify older adult fallers. However, another study by Weiss et al. (2013) did not find any significant difference between fallers and non-fallers based on the number of steps. Secondly, most fall-risk prediction studies involve retrospective or prospective data of real-life falls collected subjectively via a fall diary. Such a method can introduce a recall bias on the number and type of falls (i.e., the cause of falls). Usually, a large sample of participants are needed to be monitored consistently over a long period of time to collect sufficient fall events. With recent advances in technology, it is possible to reproduce slip and trip-like falls that closely resemble those encountered in daily life (albeit in safe laboratory conditions) to determine the prognostic capacity of various fall-risk measures (Bhatt et al., 2011). Thus, such experimental method allows for an immediate and quantifiable investigation of participants' susceptibility to certain types (i.e., slip and trip) of falls over a short test session.

In summary, free-living steps data, an easily accessible aspect of PA, collected by smartphone could largely increase the feasibility of studies on PA in the geriatric population under realistic community-living conditions. With the ability to reproduce real-life like falls in a laboratory environment, we could explore the association between daily steps data and fall-risk without the need to conduct a longitudinal study with community-based monitoring. If any association between daily steps taken and fall-risk is established, smartphone-based PA monitoring could provide health professionals with a meaningful outcome measure, in addition to existing clinical measurements, to better identify older adults at high fall-risk.

Thus, this study examined whether smartphone-derived steps data either as a single factor or along with other commonly used clinical fall-risk measures could predict laboratory-induced slip or trip related fall-risk in older adults. We also examined if the steps data would correlate with the questionnaire-based PA assessment, the PASE, and with other commonly used clinical fall-risk measures. Additionally, the study included a sub-analysis to determine the prediction capacity of both 1-week step data vs. 1-month step data to better understand the time dependency, if any on the predictive and associative relationships of step data.

\section{METHOD}

\section{Participants}

Community-dwelling ambulatory healthy older adults were recruited within a 50-mile radius from the laboratory in the city and the neighboring suburbs of the Greater Chicago Area. The study participants were recruited through advertisements via study flyers distributed at different senior centers, community exercise centers, and independent senior living facilities. Participants were included in the study if they were at least 60 years old, weighed $<250$ pounds, received a cognitive score
TABLE 1 | Sample demographics and baseline clinical measurements with the mean and standard deviations.

\begin{tabular}{lcc}
\hline Variables & Fallers $(\boldsymbol{N}=\mathbf{3 6})$ & Non-fallers $(\mathbf{N}=\mathbf{1 3})$ \\
\cline { 2 - 3 } & Mean (SD) & Mean (SD) \\
\hline Age (y) & $71.72(5.56)$ & $66.92(5.15)$ \\
Weight (lbs) & $160.73(30.41)$ & $152.84(30.77)$ \\
Height (m) & $1.64(0.81)$ & $1.71(0.85)$ \\
TUG (s) & $8.10(1.23)$ & $7.64(1.21)$ \\
BBS (out of 56) & $53.69(2.05)$ & $53.46(2.36)$ \\
Fall history (\%) & $47 \%$ & $38 \%$ \\
ABC (\%) & $87.80(11.47)$ & $85.16(12.55)$ \\
PASE & $129.57(62.60)$ & $159.54(70.29)$ \\
MMSE & $29.5(0.77)$ & $28.92(1.65)$ \\
1-week steps & $30534(17637.5)$ & $34286(18544.5)$ \\
1-month steps & $131528(79996.9)$ & $151004(79328.5)$ \\
- <1,00,000 steps & $62257.5(23476.2)$ & $62139(27319)$ \\
- 1,00,000-2,00,000 & $139899.6(33210)$ & $145497(26911)$ \\
- >2,00,000 steps & $270834(55744.2)$ & $256759.7(39458)$ \\
\hline
\end{tabular}

of $>25$ on the Folstein Mini Mental Status Exam (MMSE), possessed a smartphone with the "Google Fit" application for Android phones or "Health" application for iPhones for steps data collection, and if they had installed and enabled their respective application for at least 1 month prior to screening. Exclusion criteria included participants with acute ( $<6$ months) musculoskeletal conditions such as back pain or fracture or having a surgical history 6 months prior to the laboratory perturbation test. Seventy-six participants agreed to participate in this pilot study and were included in the initial screening. Participants were excluded if they did not pass the initial screening test $(n=9)$, did not have entire 1 month smartphone data $(n=8)$ or if the smartphone steps data was not recorded even if the participants mentioned that they carried their phones $(n=5)$ or had incomplete laboratory data due to missing markers $(n=5)$. Ultimately, 49 participants were included in the final analysis (Table 1). All participants provided written informed consent and this study was approved by the Institutional Review Board.

\section{Study Design, Protocol, and Outcome Variables}

On the initial screening day, all participants underwent various clinical measures to assess their balance [Berg Balance Scale (BBS)], balance confidence [Activities-specific Balance Confidence scale $(\mathrm{ABC})$ ], functional mobility [Timed Up-andGo test (TUG)], and PA [Physical Activity Scale for the Elderly (PASE)]. The BBS assesses balance during functional tasks, and the scores range from 0 to 56 with higher scores indicating better balance and lower fall-risk (Whitney et al., 1998; Steffen et al., 2002). The ABC scale assesses balance confidence across 16 activities, and the scores range from 0 to $100 \%$ with higher percentages indicating a higher level of balance confidence (Powell and Myers, 1995; Myers et al., 1998). The TUG score 


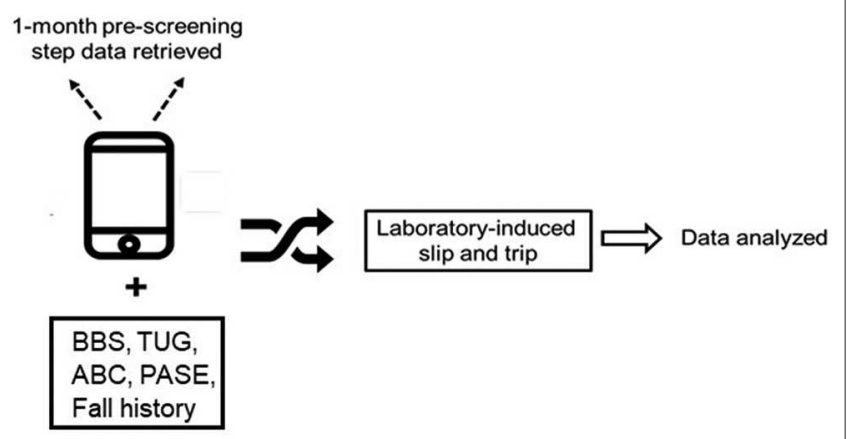

FIGURE 1 | This figure demonstrates the study protocol. Participants were subjected to various fall-risk assessment measures such as Berg Balance Scale (BBS), Activities specific balance confidence scale (ABC), Physical activity scale for elderly (PASE), and previous 1-year fall history. One-month smartphone steps data was also retrieved. Participants were subjected to various fall-risk assessment measures such as the Berg Balance Scale (BBS), Activities specific Balance Confidence scale (ABC), Timed Up and Go (TUG), Physical Activity Scale for Elderly (PASE) and previous 1-year fall history. One week and one-month smartphone steps data was also retrieved.

represents the time taken to stand up from a chair, walk a distance of 3-m, turn around, and sit back on the chair. Higher scores represent greater time taken to complete the test, indicating poor functional mobility, and high fall-risk (Shumway-Cook et al., 2000; Bohannon, 2006). The PASE scores were calculated from weights and frequency values for each of the 12 types of activities, and a higher score indicates greater PA (Washburn et al., 1993). Self-recalled fall history for the past 1 year was also obtained (Figure 1).

In addition, on the day of the initial screening the total steps for the past 1-week and 1-month were retrieved and summed from participants' smartphones. Figure 2 presents the number of steps walked each week for 4 weeks to indicate that consistent data was collected thereby depicting their consistent PA behavior. Based on the sum of 1-month step data collected for each participant, we classified the data in 3 sub-categories $(<1,00,000$ steps, 1,00,000-2,00,000 steps, and more than 2,00,000 steps). A qualitative questionnaire including four questions were asked (1) number of hours the participant carries his or her phone, (2) the time of day when the participant is most active, (3) whether the participant carries his or her phone all the time they were active, and (4) whether he or she owns a wearable fitness tracker such as FitBit or Apple watch. All participants were then scheduled to receive the laboratory slip and trip perturbations within 2 weeks of the initial screening date (Figure 1).

\section{Laboratory Fall Test}

During the laboratory session, participants were assigned to receive a novel slip and trip perturbation in random order. Participants first had to walk 25-35 unperturbed baseline trials to become familiar with the laboratory walking environment before receiving their novel slip or trip perturbation. Each participant received a single slip and a single trip given in a random order. Thus, the participants experienced a total of two

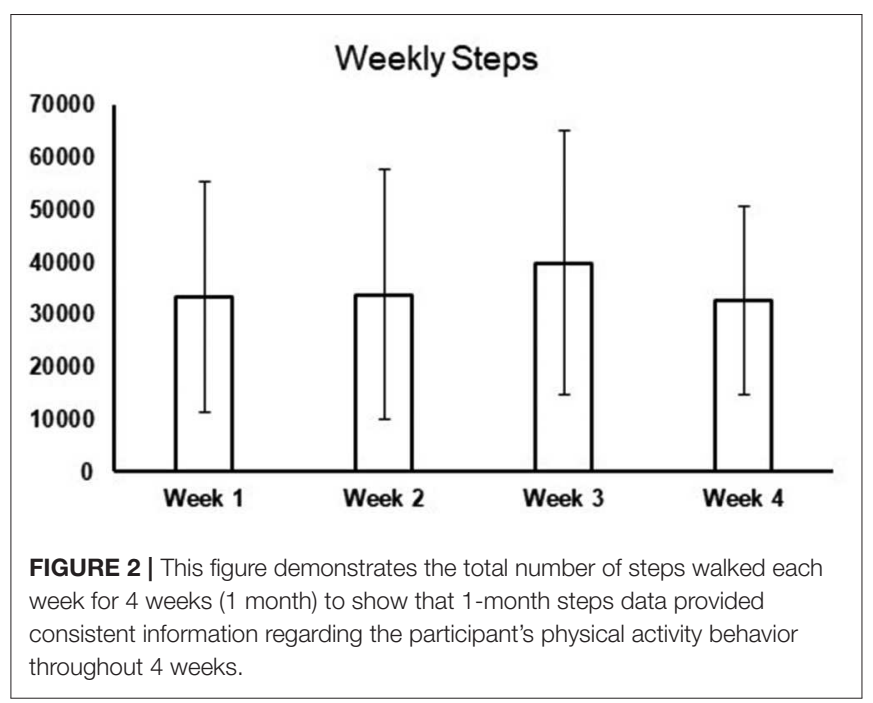

perturbations while undergoing the laboratory test. They were informed "a slip or trip may or may not occur during walking." The starting position was adjusted during baseline walking to ensure the upcoming slip or trip trials were induced properly. The slip was induced by a pair of low-friction movable platforms imbedded in a 7-meter walkway. These platforms were mounted on top of low-friction aluminum tracks resting on four force plates (AMTI, Newton, MA). The unannounced release of the platform occurred at heel strike of the perturbed (right) limb, and, following the platform's release, it was free to slide a distance of up to $60 \mathrm{~cm}$ (Wang et al., 2019a). Such slip distance has been reported to be enough to induce a fall in older adults (Figure 3A). The laboratory trip was induced on the left side by a hinged metal plate imbedded in the same walkway. During regular walking, the plate was locked in a flat position by a pair of electromagnets. For the trip trial, the electromagnets that kept the metal plate in a flat position were powered off to unlock the plate and the springs returned the plate to an upright position to induce a trip when the vertical ground reaction force (GRF) under the unperturbed (right) limb exceeded $80 \%$ of the participant's body weight after right heel strike (Figure 3B; Wang et al., 2012). All participants were protected by a safety harness connected through a load cell (Transcell Technology Inc., Buffalo Grove, IL) to a lowfriction trolley-and-beam system mounted to the ceiling along the walking path. A fall was determined if the load cell detected more than $30 \%$ of the participant's body weight after the slip or trip (Yang and Pai, 2011). Additionally, the perturbation outcome was determined to be a fall based on the video recording if the participant was visually observed to have fallen after the novel slip or trip.

\section{Statistical Analysis}

Data was summarized using descriptive statistics (means and standard deviations) for all variables including demographics, that is, age, height and weight, fall-risk measurements such as BBS, TUG, ABC, PASE as well as previous 1-year fall history and 1 -week and 1-month smartphone steps. In addition, means and 
standard deviations were also calculated for each sub-category based on 1-month data $(<1,00,000$ steps, $1,00,000-2,00,000$ steps, and more than 2,00,000 steps). Paired $t$-tests were performed between faller and non-faller groups for overall 1-week and 1-month step data as well as its subcategories to determine whether there is a significant difference in step data between fallers and non-fallers (Lee et al., 2019). Multiple univariate logistic regression analyses were performed to individually identify variables that could best predict laboratory fall-risk (induced by a slip and trip trial) in older adults. Laboratory fall outcome was treated as a binominal variable with the outcome for participants who fell in either one or both of the two perturbations being denoted as 1 or else with a 0 . Hours of phone carriage was also inputted into the logistic regression as a covariate. Based on the univariate logistic regression results, variables with a significance of $\leq 0.1$ were included in the multivariate logistic regression analysis (Bursac et al., 2008; Sperandei, 2014). A multivariate logistic regression analysis using a backward stepwise method was performed to generate a model with variables that could best predict laboratory-induced slip or trip induced falls in older adults. A receiver operating characteristic (ROC) curve was used to determine the cut-off scores (a score with the highest sensitivity and specificity) of significant variables in the univariate logistic regression and to determine the area under the curve (AUC) for the overall model predicted by the multivariate logistic regression. Pearson correlations were conducted to examine the relationships between participants' demographics, total 1-week and 1-month smartphone collected steps, fall-risk measurements, and PASE. Point biserial correlation was applied to examine relationships between steps data and the dichotomous fall histories.

\section{RESULTS}

Based on the results of the qualitative questionnaire, 12 older adults used Android phones whereas 37 used an iPhone. On average, participants carried their phones $9 \mathrm{~h}(9.06 \pm 5.6 \mathrm{~h})$ per day. Of all the participants, 38 reported they were active most of the time they carried their phones, 6 reported they were active even during the time they did not carry their phones, and 5 were unable to recall or answer the question. Only 11 participants owned a wearable device such as a Fitbit.

Out of the 49 participants, 35 participants fell on at least one perturbation during the laboratory fall test. Thirty-two participants fell only on the slip perturbation, 18 participants fell only on the trip perturbation, and 14 participants fell on both slip and trip perturbations. Table 1 indicates the means of

TABLE 2 | Variables and their significance ( $p$-value) and $R$ square value based on univariate logistic regression results.

\begin{tabular}{lcc}
\hline Variable & $\boldsymbol{p}$-value & $\boldsymbol{R}$ square value \\
\hline Age & 0.011 & 0.147 \\
Weight & 0.592 & 0.006 \\
Fall History & 0.056 & 0.075 \\
MMSE & 0.177 & 0.036 \\
ABC & 0.313 & 0.022 \\
BBS & 0.820 & 0.001 \\
TUG & 0.043 & 0.100 \\
PASE & 0.320 & 0.020 \\
1-week steps & 0.863 & 0.003 \\
1-month steps & 0.198 & 0.040
\end{tabular}

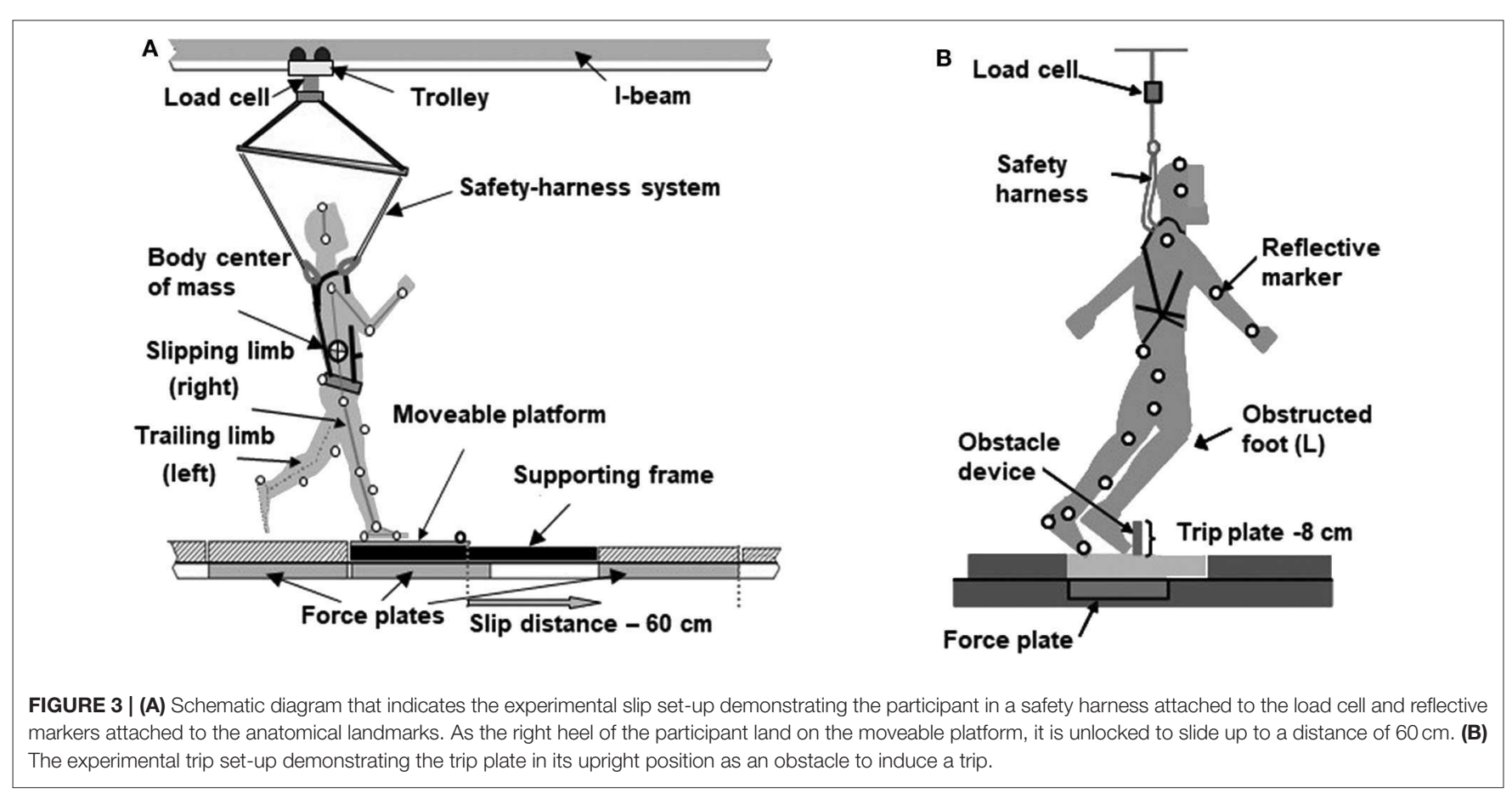


TABLE 3 | Overall model predicted based on multivariate logistic regression results along with the sensitivity, specificity, overall accuracy, and the area under the curve $(A \cup C)$ found using the Receiver Operating Curve (ROC).

\begin{tabular}{|c|c|c|c|c|c|c|c|}
\hline Model & Variable & Significance & Sensitivity & Specificity & Overall accuracy & Overall significance & AUC \\
\hline \multirow[t]{2}{*}{1} & Age & 0.006 & 97.1 & 41.7 & 83.0 & 0.002 & 0.807 \\
\hline & Fall history & 0.0065 & & & & & \\
\hline \multirow[t]{3}{*}{2} & Age & 0.012 & 94.3 & 58.3 & 85.1 & 0.002 & 0.831 \\
\hline & TUG & 0.169 & & & & & \\
\hline & Fall history & 0.122 & & & & & \\
\hline
\end{tabular}

demographic data and outcome measures of fallers and nonfallers groups. Based on the results of the paired $t$-test there was no significant difference in overall 1-week and 1-month step data or its sub-categories between fallers and non-fallers $(p>0.05)$. Based on the univariate regression analyses, age, and TUG were the only significant variables with the significance value set at $p$ $=0.05$ (Table 2 ) and fall history having a near significant value of $p=0.056$. Based on the results of the ROC curve, we established that the variable age with a cut-off score of 69.5 had a sensitivity of $63.9 \%$ and specificity of $61.5 \%$, indicating that older adults above 69.5 years had a greater fall-risk. Similarly, for the variable TUG, the cut off score of 7.49 had a sensitivity of $80 \%$ and specificity of $58.3 \%$, indicating that older adults who took longer than $7.49 \mathrm{~s}$ to complete the TUG test were at greater fall-risk.

Furthermore, variables with a significance of $\leq 0.1$ for the univariate logistic regression analysis were included in the multivariate logistic regression analysis using the backward stepwise method (Table 2). The multivariate regression analysis revealed an overall model (Model 1) including age and fall history with an overall accuracy of $83 \%$ to predict laboratory-induced falls with a sensitivity of $97.1 \%$ and specificity of $41.7 \%$ ( $p=$ 0.002 ). The area under the curve (AUC) for the model was 0.807 . A model before the final model included TUG in addition to variables age and fall history (Model 2). Addition of TUG in the final model (Model 2) improved the overall accuracy to $85.1 \%$ with a sensitivity of $94.3 \%$ and a better specificity of $58.3 \%(p$ $=0.002$ ). The AUC of this model increased to 0.831 (Table 3). Figure 4 indicates the AUC for both the models.

Thus, participants who were older, had a higher TUG score (took longer to complete the test), and had a history of falls were more likely to fall during the laboratory-induced perturbations. The best logistic regression model which predicted immediate laboratory fall is represented as Predicted Logic of (Laboratory fall $)=18.175+(0.207)^{*}$ Age $+(0.525)^{*}$ TUG $+(1.324)^{*}$ History of fall. Based on this, the odds of falling for a person who had a fall history in the previous year would be 3.75 times more than those with no falls. Similarly, for each $1 \mathrm{~s}$ increase in TUG score (slower), the odds of experiencing perturbation-induced falls would be 1.69 times higher. Further, for every 1-year increase in age, the odds of falling on laboratory perturbation would be 1.22 times higher.

Pearson correlations revealed a significant negative correlation between age and hours of phone carriage $(r=$ $-0.300, p=0.046$ ), indicating that the older participants carried their phones for less time than the younger ones. One-week steps

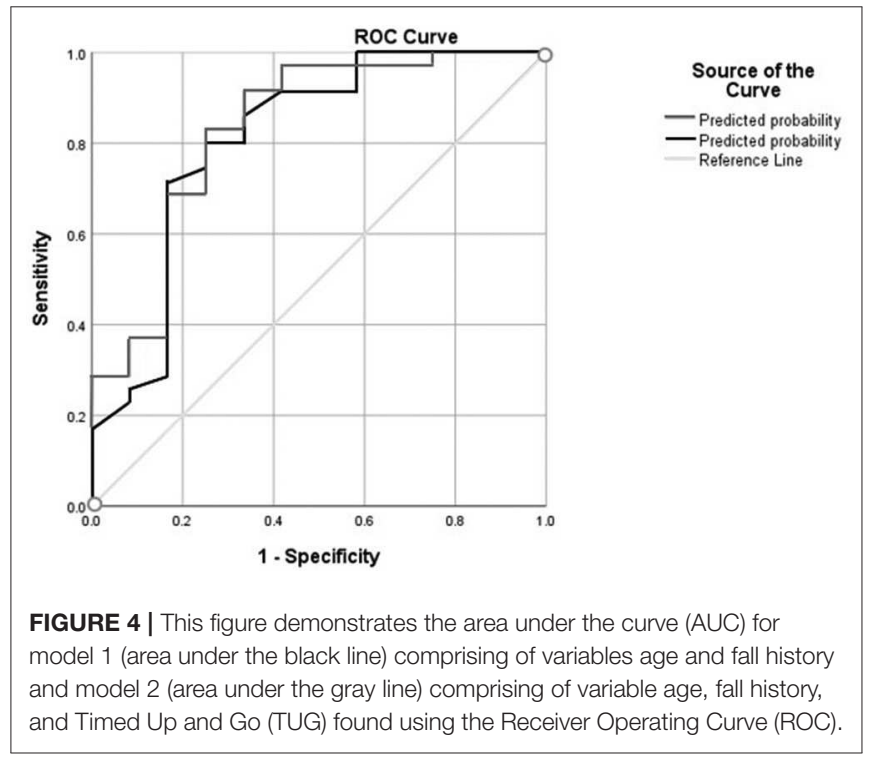

data did not correlate with any clinical fall-risk measure and history of fall. However, 1-month steps data positively correlated with higher BBS $(r=0.386, p=0.006)$ and ABC $(r=0.369, p$ $=0.012$ ) scores and negatively correlated with previous 1-year fall history $\left(r_{\mathrm{pb}}=-0.293, p=0.041\right)$. Additionally, hours of phone carriage positively correlated with steps data $(r=0.327$, $p=0.028$ ) and, hence, was inputted as a covariate in logistic regression. There was no significant correlation between steps data and PASE score.

\section{DISCUSSION}

This study explored the relationship between smartphone steps data and other commonly used clinical fall-risk measures and determined their ability to predict laboratory-induced slip or trip falls in healthy community-dwelling older adults. Univariate logistic regression analyses predicted age and TUG as individual significant predictors of laboratory falls with fall history having a near significance value. Multivariate logistic regression determined a model, which included age and fall history that best predicted laboratory-induced slip or trip fallrisk. Addition of TUG to the final model improved the overall prediction capacity of the model. Neither 1-week nor 1-month 
steps data could predict laboratory-induced fall-risk as a single predictor or in the multivariate model. A weak but significant positive correlation was noted between 1-month smartphone steps and $\mathrm{BBS}$ and $\mathrm{ABC}$ scores and a negative correlation was seen with previous 1-year fall history. No correlation was found between smartphone steps and PASE scores or other fall-risk measures.

Based on the multivariate logistic regression results, the final model predicted included age and fall history (Model 1) with a sensitivity of $97.1 \%$, specificity of $47.1 \%$, overall accuracy of $83 \%$, and AUC of 0.807 , indicating that older adults with a previous history of fall were predisposed to a greater fallrisk during laboratory-induced perturbations. Our results are consistent with the previous literature indicating that older adults with a fall history have difficulty maintaining postural control and thus are at a higher fall-risk (Horak et al., 1989; Ambrose et al., 2013). Similarly, the relationship between aging and falls is consistent with previous literature which indicated that fall rates increase with aging (Ageing Life Course family Community Health World Health Organization, 2008). For example, Pai et al. (2010) demonstrated reduced stability control in older adults compared to young adults, thereby predisposing them to a greater risk for falls during laboratory-induced slip perturbations. Furthermore, aging also has an effect on the recovery stepping response which is critical for establishing a new functional base of support following a perturbation thereby further increasing fall-risk (Tseng et al., 2009).

A model before the final model included TUG in addition to variables age and fall history (Model 2) with similar sensitivity of $94.3 \%$, a better specificity of $58.3 \%$, overall accuracy of $85.1 \%$, and a higher AUC of 0.831. Additionally, TUG as a single factor had significant prediction for laboratory-induced falls, indicating that participants who took longer to finish the test had greater risk for laboratory-induced falls. Our results were similar to a previous study, wherein TUG was able to independently predict $60 \%$ of slip-induced falls (Bhatt et al., 2011), and these falls resulted in the center of mass (COM) moving behind the forwardly sliding base of support (BOS). Thus, a lower TUG score could indicate a superior ability of the participant to rapidly relocate the COM over the displaced BOS resulting in improved COM state stability against slip-induced balance loss (Pai and Iqbal, 1999). Conversely, participants would experience forward instability following a trip perturbation due to the forward shift of both COM velocity and displacement with respect to the BOS. Therefore, a faster walking speed could increase forward instability, resulting in a greater risk for trip-induced falls (Pavol et al., 2001; Wang et al., 2019b). The TUG test scores could thus have had opposing predictive effects for slip vs. trip perturbation. In spite of the possibility of such an opposing relationship between TUG scores and fall-type, TUG emerged as a significant fall-risk predictor probably due to the fact that there were greater slip falls $(n=32)$ than trip falls $(n=18)$. However, in spite of being a significant predictor in the univariate regression analyses, it was not included in the final multivariate logistic regression model comprising of age and fall history probably due to the opposite effect of walking speed on the recovery of slip and trip explained above (Model 1). Even though TUG was included in the second model, surprisingly the individual significance of the variables fall history and TUG was lost. This might possibly be because in Multivariate logistic regression analysis, the two variables are basically "competing" with each other for explaining laboratory falls.

Although smartphones are prevalent and can be readily used in the community, steps data collected were unable to accurately predict laboratory falls. Additionally, our results indicated no difference in total 1 -week, 1 month, or sub-category step data between fallers and non-fallers. Thus, indicating that either there was no association between daily steps, a single aspect of PA, and fall-risk (given the steps collected by smartphones were accurate) or smartphone is an insufficient tool in collecting steps data under free-living condition. Our study survey indicated that only $22.44 \%$ (11/49) of participants in the current study had access to an extra wearable PA tracker (Fitbit). Thus, older adults majorly relied on smartphones for PA monitoring. However, a few study participants mentioned in the survey that they only carried their phones when going outside. Thus, we might have missed out on data when older adults were physically active but not carrying their phones, especially when they were walking inside their homes while performing daily routine activities. Moreover, participants were not given any instruction by the experimenter on the way they should carry their smartphone. Thus, the participants could have carried their smartphones in many different manners, and the phones could have recorded steps differently based on their position and orientation (Carter et al., 2018; Funk and Karabulut, 2018). A recent study also suggested that smartphone updates and different application versions might potentially change the outcomes of smartphonebased assessments (Brodie et al., 2018). It is also unclear whether the type of smartphone application matters, and if there was a difference between the "Health" and "Google Fit" applications since both were used in the study.

PA assessed using PASE was not a significant predictor of fall-risk. The PASE questionnaire might not accurately predict fall-risk in older adults as it only monitors PA over a span of 7 days, which might not be enough to provide an overall view of older adults' PA. As mentioned earlier, recall bias involved in such self-report technique might also limit the accuracy of PA measurement. Additionally, studies have demonstrated reporting bias for subjective questionnaires like PASE, such as individuals overestimating the time spent on strenuous activities or underestimating the time spent on activities that require less exertion (Bolszak et al., 2014). Also, such subjective questionnaires might lead to participants giving socially desirable answers. As PA is encouraged in older adults, participants might overestimate their PA to attain social approval. Such behavior would provide inaccurate data, thus further limiting PASE's sensitivity for fall prediction. Furthermore, PASE involves scoring an individual based on their frequency scores obtained for moderate to strenuous activities which may not be commonly performed by older adults. Thus, PA assessments should comprise of activities performed frequently by older adults to provide better fall-risk prediction. Measurement of these activities might also explain why PASE scores did not correlate with smartphone steps, as PASE considered the overall PA of 
older adults, including everyday household, recreational, and occupational activities, whereas smartphones only considered steps data.

BBS was not a significant predictor of falls in healthy older adults, which is consistent with the previous literature (Mancini and Horak, 2010; Bhatt et al., 2011). Previous studies suggest that individuals with a BBS cut-off score of 45 and above are high-functioning and at a lower risk of falls (Berg et al., 1992a, 1997). However, despite the average BBS score in our study sample being 53.57, a score much higher than the threshold suggesting a low risk of fall (Berg et al., 1992b), 36 participants out of 49 still fell during at least one laboratory-induced perturbation and 22 had previous fall histories, indicating the limited sensitivity of BBS. One probable reason might be that BBS assesses volitional balance control and does not account for or measure impairments in reactive balance. Secondly, it has shown to have a ceiling effect in healthy older adult population as it rates performance mostly during standing tasks (Newton, 1997; Langley and Mackintosh, 2007). Such tasks might not be challenging enough to assess dynamic balance control during daily living functional tasks in our population of communitydwelling, healthy older adults. Thus, the tasks performed and tested in BBS lack task-specificity to assess fall-resisting skills, thereby indicating its limited sensitivity for predicting fall-risk upon exposure to real-life like large external perturbations.

Psychosocial factors assessed in terms of balance confidence and fear of falling are crucial for fall prediction. However, balance confidence measured using $\mathrm{ABC}$ was not a significant fall-risk predictor. Previous studies done using $\mathrm{ABC}$ showed inconsistent results for ABC's ability to predict fall-risk (Lajoie and Gallagher, 2004; Schepens et al., 2010). While few studies demonstrated that $\mathrm{ABC}$ scores could differentiate fallers from non-fallers, with fallers having a lower ABC score (Mak and Pang, 2009; Hadjistavropoulos et al., 2011), other studies did not demonstrate a link between $\mathrm{ABC}$ scores and falls. The study results indicate the mean $\mathrm{ABC}$ score for our study population was $85.79 \%$. However, despite having a high balance confidence score, suggesting a low risk of fall, over half of participants fell upon experiencing a slip or trip perturbation, indicating that $\mathrm{ABC}$ might demonstrate a ceiling effect as individuals may overestimate their balance abilities. Thus, there could be a potential mismatch between individuals' self-perception of their own balance abilities and their actual functional mobility, balance, and gait impairment, thus limiting ABC's sensitivity for fall prediction.

Our results indicated no correlation between 1-week steps data and commonly used clinical measurements for risk factors of falls, however a positive correlation was noted between 1-month steps data and BBS and ABC scores and a negative correlation with previous 1-year fall history. The correlation of step data with BBS could be expected considering the close association between mobility and stability and the BBS known to be a gold standard for assessing balance control in the older adults (Berg et al., 1992a; Santos et al., 2011). Previous studies have reported that enhancing one's mobility via isolated walking programs improves static and dynamic balance as well as overall postural stability (Brooke-Wavell et al., 1998; Paillard et al., 2004). Although there was a moderate positive correlation between these two variables, both variables were not selected as fall-risk predictors as discussed above.

The correlation between the ABC scale could also be expected and justified. It is known that any improvements in balance and stability may aid in reducing both fall-risk and the subsequent fear of falling in older adults (Gusi et al., 2012). Previous studies suggested that increased walking is associated with good balance perception (Yang and Hsu, 2010). As the ABC scale determines a person's own perception of balance activities, those who were more ambulatory and had more steps could have had an enhanced balance perception of themselves and vice versa. This might explain the paralleled finding of the positive relationship between steps and balance confidence. However, with the correlation between steps data and BBS and ABC being very modest $(r<0.3)$, the potential of smartphones to be used as a screening tool to identify older adults with reduced balance and balance confidence needs further investigation.

Additionally, the results of the sub-analysis found that longterm step monitoring yielded better association with commonly used clinical measures. Several studies have utilized and suggested that short-term monitoring for 1 week is adequate for PA monitoring and fall-risk (Tudor-Locke et al., 2005; Huberty et al., 2015). For example, a study done using wearable sensors in middle-aged and older women indicated that $24 \mathrm{~h}$ monitoring over a span of 1 week is a feasible approach for monitoring activity behavior (Huberty et al., 2015). However, there are several studies indicating that collecting long-term baseline data might be more accurate in yielding stochastic predictions (Mathie et al., 2004; Yang and Hsu, 2010). For example, a review article on PA monitoring suggested that long term monitoring could enable better understanding of PA behavior (Taraldsen et al., 2012). It is postulated that long-term data collection enables monitoring of day to day variability thereby providing a better understanding of consistent and habitual steps data of older adults and could thus show a better correlation with clinical fall-risk measures. While 1 -week monitoring might be more feasible and could increase compliance, our results similar to few other studies suggest that 1 -month monitoring might yield better results.

\section{STUDY LIMITATIONS}

This study has several limitations. In the current study, the results could have been affected based on the hours and ways of phone carriage by participants as no strict instruction was given regarding phone usage. Further the software applications inbuilt or installed on the phone varied (e.g., iPhone vs. Android) which could have affected the step data accuracy. However, these factors could not be controlled due to the design of the study aiming at maximally collecting data in a natural manner and environment. Future studies could conduct studies with a uniform type of hardware (smartphone) and software (application) and additionally use wearable motion sensors to validate the association between daily steps data and older adults' fall-risk in response to laboratory-induced, real-life like external environmental perturbations. Lastly, study participants were among the healthiest community-dwelling older adults 
with a good physical performance and scores on clinical measurements. Hence, it is unknown whether current findings would also apply to frail older adults who are more susceptible to falls.

\section{CONCLUSION}

The study revealed no association between smartphone steps data and laboratory fall-risk in a group of community-dwelling older adults with good physical performance. However, being the first of its kind, the current results could be leveraged to design further studies intending to use smartphone step data for fall-risk prediction. Further, the study reinforced previous findings that, older participants with fall histories and higher TUG scores were more likely to fall in the laboratory.

\section{DATA AVAILABILITY STATEMENT}

The raw data supporting the conclusions of this article will be made available by the authors, without undue reservation.

\section{REFERENCES}

Ageing and Life Course family and Community Health World Health Organization (2008). WHO Global Report on Falls Prevention in Older Age. World Health Organization.

Ambrose, A. F., Paul, G., and Hausdorff, J. M. (2013). Risk factors for falls among older adults: a review of the literature. Maturitas 75, 51-61. doi: 10.1016/j.maturitas.2013.02.009

Berg, K. O., Maki, B. E., Williams, J. I., Holliday, P. J., and Wood-Dauphinee, S. L. (1992a). Clinical and laboratory measures of postural balance in an elderly population. Archiv. Phys. Med. Rehabil. 73, 1073-1080.

Berg, K. O., Wood-Dauphinee, S. L., Williams, J. I., and Maki, B. (1992b). Measuring balance in the elderly: validation of an instrument. Can. J. Public Health. 83, S7-S11.

Berg, W. P., Alessio, H. M., Mills, E. M., and Tong, C. (1997). Circumstances and consequences of falls in independent community-dwelling older adults. Age Ageing 26, 261-268. doi: 10.1093/ageing/26.4.261

Bhatt, T., Espy, D., Yang, F., and Pai, Y. C. (2011). Dynamic gait stability, clinical correlates, and prognosis of falls among community-dwelling older adults. Arch Phys Med Rehabil. 92, 799-805. doi: 10.1016/j.apmr.2010. 12.032

Bohannon, R. W. (2006). Reference values for the timed up and go test: a descriptive meta-analysis. J. Geriatr. Phys. Ther. 29, 64-68. doi: 10.1519/00139143-200608000-00004

Bolszak, S., Casartelli, N. C., Impellizzeri, F. M., and Maffiuletti, N. A. (2014). Validity and reproducibility of the Physical Activity Scale for the Elderly (PASE) questionnaire for the measurement of the physical activity level in patients after total knee arthroplasty. BMC Musculosk. Dis. 15:46. doi: 10.1186/1471-2474-15-46

Brodie, M., Pliner, E., Ho, A., Li, K., Chen, Z., Gandevia, S. C., et al. (2018). Big data vs accurate data in health research: large-scale physical activity monitoring, smartphones, wearable devices and risk of unconscious bias. Med. Hypotheses 119:32-36. doi: 10.1016/j.mehy.2018.07.015

Brodie, M. A., Lord, S. R., Coppens, M. J., Annegarn, J., and Delbaere, K. (2015). Eight-week remote monitoring using a freely worn device reveals unstable gait patterns in older fallers. IEEE Trans. Biomed. Eng. 62, 2588-2594. doi: 10.1109/TBME.2015.2433935

Brooke-Wavell, K., Athersmith, L., Jones, P., and Masud, T. (1998). Brisk walking and postural stability: a cross-sectional study in postmenopausal women. Gerontology 44, 288-292. doi: 10.1159/000022028

\section{ETHICS STATEMENT}

The studies involving human participants were reviewed and approved by Institutional Review Board at the University of Illinois at Chicago. The patients/participants provided their written informed consent to participate in this study.

\section{AUTHOR CONTRIBUTIONS}

YW, RG, and TB made substantial contributions toward conception, design, and execution of the study. YW, RG, LK, and EW organized the data and performed statistical analysis. YW, RG, TB, EW, LK, and AS contributed in drafting the manuscript and revising it to create a final version for submission. All authors contributed to the article and approved the submitted version.

\section{FUNDING}

This study was funded by the National Institutes of Health (R01 AG050672-02).

Bursac, Z., Gauss, C. H., Williams, D. K., and Hosmer, D. W. (2008). Purposeful selection of variables in logistic regression. Sour. Code Biol. Med. 3:17. doi: 10.1186/1751-0473-3-17

Carpenter, C. R., Cameron, A., Ganz, D. A., and Liu, S. (2019). Older adult falls in emergency medicine: 2019 update. Clin. Geriatr. Med. 35, 205-219. doi: 10.1016/j.cger.2019.01.009

Carter, D. D., Robinson, K., Forbes, J., and Hayes, S. (2018). Experiences of mobile health in promoting physical activity: a qualitative systematic review and meta-ethnography. PLoS ONE 13:e0208759. doi: 10.1371/journal.pone.0208759

Chan, B. K., Marshall, L. M., Winters, K. M., Faulkner, K. A., Schwartz, A. V., and Orwoll, E. S. (2007). Incident fall risk and physical activity and physical performance among older men: the Osteoporotic Fractures in Men Study. Am. J. Epidemiol. 165, 696-703. doi: 10.1093/aje/kwk050

Crenshaw, J. R., Bernhardt, K. A., Achenbach, S. J., Atkinson, E. J., Khosla, S., Kaufman, K. R., et al. (2017). The circumstances, orientations, and impact locations of falls in community-dwelling older women. Arch. Gerontol. Geriatr. 73, 240-247. doi: 10.1016/j.archger.2017.07.011

Duray, M., and Genc, A. (2017). The relationship between physical fitness and falling risk and fear of falling in community-dwelling elderly people with different physical activity levels. Turk. J. Med. Sci. 47, 455-462. doi: 10.3906/sag-1511-101

Ferrari, M., Harrison, B., Rawashdeh, O., Rawashdeh, M., Hammond, R., and Maddens, M. (2012). A pilot study testing a fall prevention intervention for older adults: determining the feasibility of a five-sensor motion detection system. J. Gerontol. Nurs. 38, 13-16. doi: 10.3928/00989134-20111206-01

Funk, M. D., and Karabulut, M. (2018). Smartphone carrying location and accuracy of popular pedometer smartphone apps while jogging: 1277 Board\# 85 May 31900 AM-1030 AM. Med. Sci. Sports Exerc. 50, 300-301. doi: 10.1249/01.mss.0000536071.25566.76

Gomersall, S. R., Ng, N., Burton, N. W., Pavey, T. G., Gilson, N. D., and Brown, W. J. (2016). Estimating physical activity and sedentary behavior in a freeliving context: a pragmatic comparison of consumer-based activity trackers and actigraph accelerometry. J. Med. Int. Res. 18:e239. doi: 10.2196/jmir.5531

Gusi, N., Adsuar, J. C., Corzo, H., del Pozo-Cruz, B., Olivares, P. R., and Parraca, J. A. (2012). Balance training reduces fear of falling and improves dynamic balance and isometric strength in institutionalised older people: a randomised trial. J. Physiother. 58, 97-104. doi: 10.1016/S1836-9553(12)70089-9

Hadjistavropoulos, T., Delbaere, K., and Fitzgerald, T. D. (2011). Reconceptualizing the role of fear of falling and balance confidence in fall risk. J. Aging Health 23, 3-23. doi: 10.1177/0898264310378039 
Harries, T., Eslambolchilar, P., Rettie, R., Stride, C., Walton, S., and van Woerden, H. C. (2016). Effectiveness of a smartphone app in increasing physical activity amongst male adults: a randomised controlled trial. BMC Public Health 16:925. doi: 10.1186/s12889-016-3593-9

Higgins, J. P. (2016). Smartphone applications for patients' health and fitness. Am. J. Med. 129, 11-19. doi: 10.1016/j.amjmed.2015.05.038

Horak, F. B., Shupert, C. L., and Mirka, A. (1989). Components of postural dyscontrol in the elderly: a review. Neurobiol. Aging 10, 727-738. doi: 10.1016/0197-4580(89)90010-9

Huberty, J., Ehlers, D. K., Kurka, J., Ainsworth, B., and Buman, M. (2015). Feasibility of three wearable sensors for 24 hour monitoring in middle-aged women. BMC Womens Health 15:55. doi: 10.1186/s12905-015-0212-3

Lajoie, Y., and Gallagher, S. (2004). Predicting falls within the elderly community: comparison of postural sway, reaction time, the Berg balance scale and the Activities-specific Balance Confidence (ABC) scale for comparing fallers and non-fallers. Arch. Gerontol. Geriatr. 38, 11-26. doi: 10.1016/S0167-4943(03)00082-7

Langley, F. A., and Mackintosh, S. F. (2007). Functional balance assessment of older community dwelling adults: a systematic review of the literature. Int. J. Allied Health Sci. Pract. 5, 13.

Lee, I. M., Shiroma, E. J., Kamada, M., Bassett, D. R., Matthews, C. E., et al. (2019). Association of step volume and intensity with allcause mortality in older women. JAMA Int Med. 179, 1105-1112. doi: 10.1001/jamainternmed.2019.0899

Li, F., Fisher, K. J., Brownson, R. C., and Bosworth, M. (2005). Multilevel modelling of built environment characteristics related to neighbourhood walking activity in older adults. J. Epidemiol. Commun. Health 59, 558-564. doi: 10.1136/jech.2004.028399

Logan, S. L., Gottlieb, B. H., Maitland, S. B., Meegan, D., and Spriet, L. L. (2013). The Physical Activity Scale for the Elderly (PASE) questionnaire; does it predict physical health? Int. J. Environ. Res. Public Health 10, 3967-3986. doi: 10.3390/ijerph10093967

Lu, Y., Wei, Y., Liu, L., Zhong, J., Sun, L., and Liu, Y. (2017). Towards unsupervised physical activity recognition using smartphone accelerometers. Multimed. Tools Appl. 76, 10701-10719. doi: 10.1007/s11042-015-3188-y

Luukinen, H., Herala, M., Koski, K., Honkanen, R., Laippala, P., and Kivela, S. L. (2000). Fracture risk associated with a fall according to type of fall among the elderly. Osteoporos Int. 11, 631-634. doi: 10.1007/s001980070086

Mak, M. K., and Pang, M. Y. (2009). Balance confidence and functional mobility are independently associated with falls in people with Parkinson's disease. J. Neurol. 256, 742-749. doi: 10.1007/s00415-009-5007-8

Mancini, M., and Horak, F. B. (2010). The relevance of clinical balance assessment tools to differentiate balance deficits. Eur. J. Phys. Rehabil. Med. 46, 239-248.

Marschollek, M., Rehwald, A., Wolf, K., Gietzelt, M., Nemitz, G., Meyer Zu Schwabedissen, H., et al. (2011). Sensor-based fall risk assessment-an expert 'to go'. Methods Inform. Med. 50, 420-426. doi: 10.3414/ME10-01-0040

Mathie, M. J., Coster, A. C., Lovell, N. H., Celler, B. G., Lord, S. R., and Tiedemann, A. (2004). A pilot study of long-term monitoring of human movements in the home using accelerometry. J. Telemed. Telecare 10, 144-151. doi: 10.1258/135763304323070788

Myers, A. M., Fletcher, P. C., Myers, A. H., and Sherk, W. (1998). Discriminative and evaluative properties of the activities-specific balance confidence (ABC) scale. J. Gerontol. Ser. A Biol. Sci. Med. Sci. 53, M287-M294. doi: 10.1093/gerona/53A.4.M287

Newton, R. A. (1997). Balance screening of an inner city older adult population. Arch. Phys. Med. Rehabil. 78, 587-591. doi: 10.1016/S0003-9993(97)90423-8

Oliveira, C. C., Lee, A. L., McGinley, J., Anderson, G. P., Clark, R. A., Thompson, M., et al. (2017). Balance and falls in acute exacerbation of chronic obstructive pulmonary disease: a prospective study. Copd 14, 518-525. doi: 10.1080/15412555.2017.1342232

Pai, Y.-C., Bhatt, T., Wang, E., Espy, D., and Pavol, M. J. (2010). Inoculation against falls: rapid adaptation by young and older adults to slips during daily activities. Archiv. Phys. Med. Rehabil. 91, 452-459. doi: 10.1016/j.apmr.2009.10.032

Pai, Y.-C., and Iqbal, K. (1999). Simulated movement termination for balance recovery: can movement strategies be sought to maintain stability in the presence of slipping or forced sliding? J. Biomech. 32, 779-786. doi: 10.1016/S0021-9290(99)00074-3
Paillard, T., Lafont, C., Costes-Salon, M., Riviere, D., and Dupui, P. (2004). Effects of brisk walking on static and dynamic balance, locomotion, body composition, and aerobic capacity in ageing healthy active men. Int. J. Sports Med. 25, 539-546. doi: 10.1055/s-2004-820948

Pavol, M. J., Owings, T. M., Foley, K. T., and Grabiner, M. D. (2001). Mechanisms leading to a fall from an induced trip in healthy older adults. J. Gerontol. Ser. A Biol. Sci. Med. Sci. 56, M428-M437. doi: 10.1093/gerona/56.7.M428

Pereira, C. L., Baptista, F., and Infante, P. (2014). Role of physical activity in the occurrence of falls and fall-related injuries in community-dwelling adults over 50 years old. Disabil. Rehabil. 36, 117-124. doi: 10.3109/09638288.2013.782355

Pereira, C. L., Vogelaere, P., and Baptista, F. (2008). Role of physical activity in the prevention of falls and their consequences in the elderly. Eur Rev. Aging Phys. Activity 5:51. doi: 10.1007/s11556-008-0031-8

Perell, K. L., Nelson, A., Goldman, R. L., Luther, S. L., Prieto-Lewis, N., and Rubenstein, L. Z. (2001). Fall risk assessment measures: an analytic review. J. Gerontol. A Biol. Sci. Med. Sci. 56, M761-M766. doi: 10.1093/gerona/56.12.M761

Plasqui, G., and Westerterp, K. R. (2007). Physical activity assessment with accelerometers: an evaluation against doubly labeled water. Obesity 15, 2371-2379. doi: 10.1038/oby.2007.281

Powell, L. E., and Myers, A. M. (1995). The activities-specific balance confidence (ABC) scale. J. Gerontol. Ser. A Biol. Sci. Med. Sci. 50, M28-M34. doi: 10.1093/gerona/50A.1.M28

Rispens, S. M., van Schooten, K. S., Pijnappels, M., Daffertshofer, A., Beek, P. J., and van Dieen, J. H. (2015). Identification of fall risk predictors in daily life measurements: gait characteristics' reliability and association with self-reported fall history. Neurorehabil. Neural Repair 29, 54-61. doi: $10.1177 / 1545968314532031$

Roig, M., Eng, J. J., MacIntyre, D. L., Road, J. D., FitzGerald, J. M., Burns, J., et al. (2011). Falls in people with chronic obstructive pulmonary disease: an observational cohort study. Respir Med. 105, 461-469. doi: 10.1016/j.rmed.2010.08.015

Rosenberger, M. E., Buman, M. P., Haskell, W. L., McConnell, M. V., and Carstensen, L. L. (2016). Twenty-four hours of sleep, sedentary behavior, and physical activity with nine wearable devices. Med. Sci. Sports Exerc. 48, 457-465. doi: 10.1249/MSS.0000000000000778

Rubenstein, L. Z. (2006). Falls in older people: epidemiology, risk factors and strategies for prevention. Age Ageing 35(Suppl. 2), ii37-ii41. doi: 10.1093/ageing/afl084

Sallis, J. F., and Saelens, B. E. (2000). Assessment of physical activity by self-report: status, limitations, and future directions. Res. Q. Exerc. Sport 71(Suppl. 2), S1-S14. doi: 10.1080/02701367.2000.11082780

Santos, G. M., Souza, A., Virtuoso, J. F., Tavares, G., and Mazo, G. Z. (2011). Predictive values at risk of falling in physically active and no active elderly with Berg Balance Scale. Brazil. J. Phys. Ther. 15, 95-101. doi: 10.1590/S1413-35552011000200003

Schepens, S., Goldberg, A., and Wallace, M. (2010). The short version of the Activities-specific Balance Confidence $(\mathrm{ABC})$ scale: its validity, reliability, and relationship to balance impairment and falls in older adults. Archiv. Gerontol. Geriatr. 51, 9-12. doi: 10.1016/j.archger.2009.06.003

Shumway-Cook, A., Brauer, S., and Woollacott, M. (2000). Predicting the probability for falls in community-dwelling older adults using the Timed Up and Go Test. Phys. Ther. 80, 896-903. doi: 10.1093/ptj/80.9.896

Smee, D. J., Anson, J. M., Waddington, G. S., and Berry, H. L. (2012). Association between physical functionality and falls risk in community-living older adults. Curr. Gerontol. Geriatr. Res. 2012:864516. doi: 10.1155/2012/ 864516

Sperandei, S. (2014). Understanding logistic regression analysis. Biochem. Med. 24 12-18. doi: 10.11613/BM.2014.003

Steffen, T. M., Hacker, T. A., and Mollinger, L. (2002). Age-and gender-related test performance in community-dwelling elderly people: Six-Minute Walk Test, Berg Balance Scale, Timed Up and Go Test, and gait speeds. Phys. Ther. 82, 128-137. doi: $10.1093 / \mathrm{ptj} / 82.2 .128$

Taraldsen, K., Chastin, S. F., Riphagen, I. I., Vereijken, B., and Helbostad, J. L. (2012). Physical activity monitoring by use of accelerometer-based body-worn sensors in older adults: a systematic literature review of current knowledge and applications. Maturitas 71, 13-19. doi: 10.1016/j.maturitas.2011.11.003 
Tseng, S.-C., Stanhope, S. J., and Morton, S. M. (2009). Impaired reactive stepping adjustments in older adults. J. Gerontol. Ser. A Biomed. Sci. Med. Sci. 64, 807-815. doi: 10.1093/gerona/glp027

Tudor-Locke, C., Burkett, L., Reis, J., Ainsworth, B., Macera, C., and Wilson, D. (2005). How many days of pedometer monitoring predict weekly physical activity in adults? Prevent. Med. 40, 293-298. doi: 10.1016/j.ypmed.2004.06.003

van Schooten, K. S., Pijnappels, M., Rispens, S. M., Elders, P. J., Lips, P., and van Dieen, J. H. (2015). Ambulatory fall-risk assessment: amount and quality of daily-life gait predict falls in older adults. J. Gerontol. Ser. A Biomed. Sci. Med. Sci. 70, 608-615. doi: 10.1093/gerona/glu225

Wang, T.-Y., Bhatt, T., Yang, F., and Pai, Y.-C. (2012). Adaptive control reduces trip-induced forward gait instability among young adults. J. Biomech. 45, 1169-1175. doi: 10.1016/j.jbiomech.2012.02.001

Wang, Y., Bhatt, T., Liu, X., Wang, S., Lee, A., Wang, E., et al. (2019a). Can treadmill-slip perturbation training reduce immediate risk of over-ground-slip induced fall among community-dwelling older adults? J. Biomech. 84, 58-66. doi: 10.1016/j.jbiomech.2018.12.017

Wang, Y., Wang, S., Bolton, R., Kaur, T., and Bhatt, T. (2019b). Effects of taskspecific obstacle-induced trip-perturbation training: proactive and reactive adaptation to reduce fall-risk in community-dwelling older adults. Aging Clin. Exp. Res. 32, 893-905. doi: 10.1007/s40520-019-01268-6

Washburn, R. A., Smith, K. W., Jette, A. M., and Janney, C. A. (1993). The Physical Activity Scale for the Elderly (PASE): development and evaluation. J. Clin. Epidemiol. 46, 153-162. doi: 10.1016/0895-4356(93) 90053-4

Weiss, A., Brozgol, M., Dorfman, M., Herman, T., Shema, S. R., Giladi, N., et al. (2013). Does the evaluation of gait quality during daily life provide insight into fall risk? A novel approach using 3-day accelerometer recordings. Neurorehabil. Neural Repair. 27, 742-752. doi: 10.1177/1545968313 491004
Welmer, A. K., Rizzuto, D., Laukka, E. J., Johnell, K., and Fratiglioni, L. (2017). Cognitive and physical function in relation to the risk of injurious falls in older adults: a population-based study. J. Gerontol. A Biol. Sci. Med. Sci. 72, 669-675. doi: 10.1093/gerona/glw141

Whitney, S. L., Poole, J. L., and Cass, S. P. (1998). A review of balance instruments for older adults. Am. J. Occupat. Ther. 52, 666-671. doi: 10.5014/ajot.52.8.666

Yang, C. C., and Hsu, Y. L. (2010). A review of accelerometry-based wearable motion detectors for physical activity monitoring. Sensors 10, 7772-7788. doi: 10.3390/s100807772

Yang, F., and Pai, Y.-C. (2011). Automatic recognition of falls in gaitslip training: harness load cell based criteria. J. Biomech. 44, 2243-2249. doi: 10.1016/j.jbiomech.2011.05.039

Young, W. R., and Mark Williams, A. (2015). How fear of falling can increase fallrisk in older adults: applying psychological theory to practical observations. Gait Posture 41, 7-12. doi: 10.1016/j.gaitpost.2014.09.006

Zhao, Y. L., Alderden, J., Lind, B. K., and Kim, H. (2018). A Comprehensive assessment of risk factors for falls in community-dwelling older adults. $J$. Gerontol. Nurs. 44, 40-48. doi: 10.3928/00989134-20180913-04

Conflict of Interest: The authors declare that the research was conducted in the absence of any commercial or financial relationships that could be construed as a potential conflict of interest.

Copyright (C) 2020 Wang, Gangwani, Kannan, Schenone, Wang and Bhatt. This is an open-access article distributed under the terms of the Creative Commons Attribution License (CC BY). The use, distribution or reproduction in other forums is permitted, provided the original author(s) and the copyright owner(s) are credited and that the original publication in this journal is cited, in accordance with accepted academic practice. No use, distribution or reproduction is permitted which does not comply with these terms. 